\title{
ON THE UNIQUENESS OF SOLUTIONS OF CERTAIN IMPROPERLY POSED PROBLEMS ${ }^{1}$
}

\author{
VINCENT G. SIGILLITO
}

\begin{abstract}
Conditions are derived for uniqueness of solutions of some improperly posed problems for hyperbolic equations. Uniqueness results for backward parabolic problems are also included.
\end{abstract}

In [1] Dunninger and Zachmanoglou derived a condition for uniqueness of the Dirichlet problem for a hyperbolic equation in cylindrical domains. The purpose of this note is to point out that the technique used in [1] can be employed, with only slight changes, to derive similar uniqueness conditions for a variety of boundary value problems for hyperbolic equations. We also indicate how the arguments of [1] can be modified to give elegant proofs of known uniqueness theorems for three initial-boundary value problems associated with the backward heat equation.

Related results were obtained in [2] for the Dirichlet and Neumann problems associated with the $n$-dimensional wave equation in rectangular domains bounded by hyperplanes normal to the coordinate axis using a variation of the methods of Bourgin and Duffin [3]. Studies concerned with questions of uniqueness, existence and continuous dependence of the solution on the boundary data have been carried out by John [4] and Fox and Pucci [5] for the two-dimensional wave equation.

Preliminaries. Denote by $B$ a bounded domain in $n$-dimensional space $R_{n}$ and by $D$ the $n+1$-dimensional cylindrical domain

$$
D=B \times I,
$$

where $I$ is the interval $0<t<T$. By $S$ and $B_{T}$ we denote the lateral surface and top of the cylinder, respectively. The following notation will be used:

$$
D_{\tau}=D \cap\{\tau<t<T\}, \quad S_{\tau}=S \cap\{\tau<t<T\}, \quad B_{\tau}=D \cap\{t=\tau\} .
$$

Received by the editors July 22, 1969.

AMS Subject Classifications. Primary 3535, 3552, 3555, 3562, 3565.

Key Words and Phrases. Improperly posed problems, uniqueness conditions for hyperbolic Neumann problem, uniqueness of solutions for backward parabolic equations.

1 This work supported by the Department of the Navy, Bureau of Naval Weapons, under Contract Now 62-0604-c. 
On the boundary $\partial D$ of $D$ the outward unit normal vector is denoted by $n=\left(n_{1}, n_{2}, \cdots, n_{n}, n_{t}\right)$. The summation convention will be used so that repeated indices are to be summed from 1 to $n$. Finally we assume that the domain $B$ is sufficiently regular to allow application of the divergence theorem and in order to ensure the existence of a complete set of sufficiently smooth eigenfunctions for the eigenvalue problems used below.

The wave equation. Assuming existence, we now derive a uniqueness condition for the Neumann problem associated with the wave equation. The proof follows closely that given in [1] with a slight modification that allows us to adopt the arguments of [1] to the Neumann problem.

Consider the homogeneous Neumann problem in $D$

$$
\begin{gathered}
L u \equiv u_{x_{i} x_{i}}-u_{t t}=0 \text { in } D, \\
\partial u / \partial n=0 \text { on } \partial D .
\end{gathered}
$$

THEOREM. Every solution of $(1,2)$ belonging to $C^{2}(D) \cap C^{1}(\bar{D})$ is unique (to within an additive constant) iff

$$
\lambda_{k}^{1 / 2} T \neq m \pi, \quad k=1,2, \cdots, \quad m=1,2, \cdots
$$

where the $\lambda_{k}$ are the nonzero eigenvalues of the eigenvalue problem

$$
\begin{gathered}
v_{x_{i} x_{i}}+\lambda v=0 \text { in } B, \\
\partial v / \partial n=0 \text { on } \partial B .
\end{gathered}
$$

Proof. The necessity follows easily for if (3) is not satisfied, then $\lambda_{k}^{1 / 2} T=m \pi$ for some positive integer $m$ and $k$. But then the function

$$
u(x, t)=v_{k}(x) \cos \lambda_{k}^{1 / 2} t,
$$

where $v_{k}$ is the eigenvalue corresponding to $\lambda_{k}$, is a nontrivial solution of $(1,2)$.

To demonstrate the sufficiency we use the integral identity.

(6) $\int_{D}(w L u-u L w) d x d t=\int_{\partial D}\left[\left(w u_{x_{i}}-u w w_{x_{i}}\right) n_{i}-\left(w u_{t}-u w_{t}\right) n_{t}\right] d \sigma$.

If $u$ satisfies $(1,2)$ and if $w(x, t)=v_{k}(x) \cos \lambda_{k}^{1 / 2} t$ then

$$
\lambda_{k}^{1 / 2} \sin \lambda_{k}^{1 / 2} T \int_{B_{T}} u(x, T) v_{k}(x) d x=0, \quad k=1,2, \cdots
$$

and by (3) this implies that 


$$
\int_{B_{T}} u(x, T) v_{k}(x) d x=0, \quad k=1,2, \cdots
$$

Since the eigenfunctions $\left\{v_{k}(x)\right\}, k=0,1,2, \cdots$, form a complete set, and $v_{0}(x)=$ constant, equation (7) implies that $u(x, T)$ is a constant.

Now integrate the identity

$$
2 u_{t} L u=2\left(u_{x_{i}} u_{t}\right)_{x_{i}}-\left(u_{x_{i}} u_{x_{i}}+u_{t}^{2}\right)_{t}
$$

over $D_{\tau}$ to obtain

$$
\begin{aligned}
2 \int_{D_{\tau}} u_{t} L u d x d t= & 2 \int_{S} u_{x_{i}} u_{t} n_{i} d \sigma+\int_{B_{\tau}}\left(u_{x_{i}} u_{x_{i}}+u_{t}^{2}\right) d x \\
& -\int_{B_{T}}\left(u_{x_{i}} u_{x_{i}}+u_{t}^{2}\right) d x
\end{aligned}
$$

Then if $u$ satisfies $(1,2)$ and $u(x, T)=$ constant, we obtain

$$
\int_{B_{\tau}}\left(u_{x_{i}} u_{x_{i}}+u_{t}^{2}\right) d x=0
$$

from which we conclude that $u$ is a constant in $D$.

REMARKS. 1. The proof goes through for the more general operator $L u \equiv\left(a^{i j} u_{x_{i}}\right)_{x_{j}}-u_{t t}$, where it is assumed that the $a^{i j}$ are functions of $x$ only, $a^{i j}=a^{j i}$, and the matrix $\left(a^{i j}\right)$ is positive definite in $B$.

2. A similar theorem can be proven with Dirichlet or mixed (i.e., $\partial u / \partial n+\alpha u)$ conditions prescribed on $S$.

3. If Dirichlet conditions are prescribed on $B$ and $B_{T}$ then a similar uniqueness condition holds for the operator $L u=\left(a^{i j} u_{x_{i}}\right)_{x_{j}}-c u-u_{\imath t}$, $c=c(x), c \geqq 0$ for either Neumann or mixed conditions given on $S$.

THE BACKWARD HEAT EQUATION. We now show how these methods can be used to give very short proofs of uniqueness for problems associated with the backward heat equation.

Consider the homogeneous Dirichlet problem in $D$

$$
\begin{gathered}
L u=u_{x_{i} x_{i}}+u+u_{t}=0 \text { in } D, \\
u=0 \text { on } S \cup B .
\end{gathered}
$$

We have the following

THEOREM. Every solution of $(8,9)$ belonging to $C^{2}(x) \cap C^{1}(t)$ in $D$ and $C^{1}(\bar{D})$ is a constant in $D$. 
Proof. We start with the integral identity

$$
\begin{aligned}
\int_{D_{\tau}}\left(w L u-u L^{*} w\right)= & \int_{S_{\tau}}\left(u \frac{\partial u}{\partial n}-u \frac{\partial w}{\partial n}\right) d \sigma \\
& +\int_{B_{\tau}} u w d x-\int_{B} u w d x
\end{aligned}
$$

where $L^{*} u=u_{x_{i} x_{i}}+u-u_{t}$. Suppose that $u$ is a solution of $(8,9)$ and $w(x, t)=v_{k}(x) e^{\lambda_{k} t}$ where $v_{k}$ is a solution of the eigenvalue problem

$$
v_{x_{\boldsymbol{i}} x_{\boldsymbol{i}}}+v+\lambda v=0 \text { in } B, \quad v=0 \text { on } \partial B .
$$

Then (10) becomes

$$
\int_{B_{\tau}} u v_{k} d x=0, \quad k=1,2, \cdots
$$

By the completeness of the eigenfunctions $\left\{v_{k}(x)\right\}_{1}^{\infty}$ this implies that $u(x, t)=0,0<t<T, x \in B$. Thus $u \equiv 0$ in $D$.

REMARKs. 1 . The proof goes through for the more general operator $L u=\left(a^{i j} u_{x_{i}}\right)_{x_{j}}+c u+u_{t}$.

2. The Dirichlet conditions on $S$ may be replaced by Neumann or mixed conditions.

\section{REFERENCES}

1. D. R. Dunninger and E. C. Zachmanoglou, The condition for uniqueness of the Dirichlet problem for hyperbolic equations in cylindrical domains, J. Math. Mech. 18 (1969), 763-766.

2. - The condition for uniqueness of solutions of the Dirichlet problem for the wave equation in coordinate rectangles, J. Math. Anal. Appl. 20 (1967), 17-21. MR 37 \#1807.

3. D. G. Bourgin and R. Duffin, The Dirichlet problem for the vibrating string equation, Bull. Amer. Math. Soc. 45 (1939), 851-858. MR 1, 120.

4. F. John, The Dirichlet problem for a hyperbolic equation, Amer. J. Math. 63 (1941), 141-154. MR 2, 204.

5. D. W. Fox and C. Pucci, The Dirichlet problem for the wave equation, Ann. Mat. Pura Appl. (4) 46 (1958), 155-182. MR 21 \#3653.

JoHNS Hopkins UNIVERSITY 